DRAFT VERSION OCTOBER 30, 2018

Preprint typeset using LTTEX style emulateapj v. 6/22/04

\title{
DO ULTRAHIGH ENERGY COSMIC RAYS COME FROM ACTIVE GALACTIC NUCLEI AND FERMI $\gamma$-RAY SOURCES?
}

\author{
YUN-YING JiANG ${ }^{1,2}$, L.G. HOU ${ }^{1}$, J.L. HAN ${ }^{1}$, X.H. SUN ${ }^{1}$, WEI WANG ${ }^{1}$ \\ Draft version October 30, 2018
}

\begin{abstract}
We study possible correlations between ultrahigh energy cosmic rays (UHECRs), observed by Auger, AGASA, and Yakutsk, and nearby active galactic nuclei (AGNs) and Fermi sources. We consider the deflection effects by a Galactic magnetic field (GMF) model constrained by the most updated measurements. We found that the average deflection angles of UHECRs by the Galactic magnetic fields are less than $4^{\circ}$. A correlation between the Auger cosmic-ray events and nearby AGNs with a significance level of $\sim 4 \sigma$ was found for the Auger UHECR data sets with or without deflection correction. No correlation was found between the AGASA/Yakutsk events with nearby AGNs. Marginal correlations between the Auger events and the Fermi sources, and between AGASA events and Fermi AGNs were found when the deflections calculated by the GMF model were considered. However, no correlation was found between the Yakutsk data and Fermi sources. Some Fermi sources are close to the arrival directions of UHECR events detected by Auger, AGASA, and Yakutsk, most of which are probably chance coincidence rather than objects producing UHECRs in the nearby Universe. Four Fermi sources, NGC 4945, ESO 323-G77, NGC 6951, and Cen A, within $100 \mathrm{Mpc}$ have UHECR events within $3^{\circ} 1$ from their positions, which could potentially be cosmic-ray accelerators. However, the association can only be confirmed if more UHECRs are preferably detected in these directions

Subject headings: (ISM:) cosmic rays - galaxies: active - magnetic field - methods: statistical
\end{abstract}

\section{INTRODUCTION}

The spectrum, origin, and composition of ultrahigh energy cosmic rays (UHECRs) with energies $\geqslant 10^{19} \mathrm{eV}(=10$ $\mathrm{EeV})$ are a long standing mystery in high-energy astrophysics (Hillas 1984). Greisen (1966) and Zatsepin \& Kuz'min (1966) showed a theoretical distant limit for the cosmic rays with energies of order $10^{20} \mathrm{eV}$ traveling through the microwave background radiation field, which is called the GZK effect. Because of the GZK effect, particles with energies above $10 \mathrm{EeV}$ are able to reach our Earth only from nearby sources within about $100 \mathrm{Mpc}$. Another barrier in the investigation of the UHECR origin is the deflections of UHECRs by the magnetic fields. Due to the poor knowledge of the extragalactic and intergalactic magnetic fields, the deflections of UHECRs have not yet understood. Dolag et al. (2004, 2005) suggested that the deflections by extragalactic magnetic fields are generally less than $1^{\circ}$, while Ryu et al. (2010) and Sigl et al. (2003) claimed that could be larger than $10^{\circ}$. The Galactic magnetic fields (GMFs) are relatively better known (e.g. Han et al. 2006; Sun et al. 2008) and are widely discussed in the studies of UHECR origin (e.g. Stanev 1997; Tinyakov \& Tkachev 2002; Prouza \& Smída 2003; Nagar \& Matulich 2009). Kachelrieß et al. (2007) concluded that the deflections of UHECRs by the GMFs cannot be neglected even for the protons of $E=10^{20} \mathrm{eV}$, since the deflection angles are comparable with the angular resolution of current experiments. Nagar \& Matulich (2009) tried seven GMF models to study the correlations between UHECRs and source population(s). However, no halo component was included in the four GMF models they used and another three GMF models adopted from Sun et al. (2008)

\footnotetext{
${ }^{1}$ National Astronomical Observatories, Chinese Academy of Sciences, 20A Datun Road, Chaoyang District, Beijing 100012, China; hjl@ nao.cas.cn

${ }^{2}$ Graduate University of the Chinese Academy of Sciences, Beijing 100049, China
}

have a strong halo component about $7 \mu \mathrm{G}$. Observational constraints on the Galactic magnetic field strength (Han \& Qiao 1994; Han et al. 1999; Morris \& Serabvn 1996) and the configuration of disk magnetic fields (Han et al. 2006; Han 2009) should be carefully considered in the GMF model.

Since the discovery of UHECRs (Linsley 1963), many equipments have been used to search for these events, including Fly's Eye (Bird et al. 1994), Yakutsk Extensive Air Showers Array (Ivanov et al. 2003a; Pravdin et al. 2005), Akeno Giant Air Shower Array (AGASA; Hayashida et al. 2000; Takeda et al. 2003), High Resolution Fly's Eye cosmic-ray detector (HiRes; Abbasi et al. 2004, 2008) and Pierre Auger Observatory (PAO; Abraham et al. 2004; The Pierre Auger Collaboration 2007, 2008). The existence of the GZK cutoff has been observed by the HiRes and Auger (Abbasi et al. 2008; The Pierre Auger Collaboration 2008). Some objects have been suggested to be possible sources of UHECRs, e.g., pulsars (Blasi et al. 2000), active galactic nuclei (AGNs) and subclasses of AGNs (Protheroe \& Szabo 1992; Farrar \& Biermann 1998; Tinvakov \& Tkachev 2001a b; Virmani et al. 2002; Gorbunov et al. 2002, 2004; Abbasi et al. 2006; Farrar et al. 2009), radio lobes of FR II galaxies (Rachen \& Biermann 1993; Hardcastle et al. 2009), and $\gamma$-ray bursts (Waxman 1995; Milgrom \& Usov 1995). However, the real sources of UHECRs are not known yet. AGNs are favored as the most probable sources for accelerating particles to the extreme energies (Hillas 1984) for a long time.

Recently, The Pierre Auger Collaboration (2007, 2008) studied the correlation between the arrival directions of UHECRs and the positions of nearby AGNs in the Véron-Cetty \& Véron (2006) AGN catalog (hereafter VCV catalog). They concluded that the arrival directions of cosmic rays with energies above $\sim 60 \mathrm{EeV}$ are anisotropic and UHECRs have a good correlation with the positions of nearby AGNs $(z<0.018)$. The intriguing result attracted 
much attention. Ivanov (2008) found the correlation between Yakutsk UHECRs and the nearby VCV AGNs $(\lesssim 100 \mathrm{Mpc})$. George et al. (2008) investigated the correlation between the Swift Burst Alert Telescope AGN catalog with the Auger UHECR events, and found a correlation at a significance level of $98 \%$ when the AGNs were weighted by their hard X-ray flux and the Auger experiment exposure. However, some associated AGNs of Auger events may not have enough energy to accelerate particles to ultrahigh energies (Moskalenko et al. 2009). The High Resolution Flys Eye Collaboration searched for possible correlation between the HiRes UHECRs and AGNs located in the northern hemisphere; however, no significant correlation was found.

The Fermi high energy $\gamma$-ray sources are also possible UHECR sources. The recently released Fermi Large Area Telescope First Source Catalog (1FGL) contains $1451 \gamma$-ray point sources (Abdo et al.2010) with nearly uniform sky coverage (Atwood et al. 2009). Mirabal \& Oya (2010) first investigated the correlation between Auger UHECRs and 1FGL sources without considering the deflection by the GMFs and redshifts of Fermi AGNs, and concluded that the UHECRs are not associated with Fermi sources. The possible correlation of UHECRs and Fermi sources should be re-examined after the GZK cutoff and the UHECR deflection by the GMFs are considered.

In this work, we construct a new GMF model based on the updated measurements of the Galactic magnetic fields and investigate the deflections of UHECRs by the GMFs. Considering the GZK cutoff and the deflection correction through our GMF model, we re-examine the possible correlation between UHECRs and nearby AGNs and Fermi sources. In Section 2, we discuss available data of UHECRs detected by Auger, AGASA, and Yakutsk and possible astrophysical objects. The deflections of UHECRs by the GMFs are discussed in Section 3. The correlation studies are given in Section 4. Discussions and conclusions are presented in Section 5.

\section{UHECR DATA AND POTENTIAL COSMIC RAY SOURCES}

\subsection{UHECR events}

High-quality UHECR data and reasonable deflection correction by the GMF models are crucial to understand the origin of UHECRs. Therefore, we only consider UHECR events which satisfy two criteria: (1) good angular and energy resolutions; (2) ultrahigh energy $(E>40 \mathrm{EeV})$ which has a predictable small deflection angle.

We work on the UHECR events recorded by Auger, AGASA, and Yakutsk. The HiRes UHECRs have a typical angular resolution of $0^{\circ} 6$ (Abbasi et al. 2004) and 27 events have been published. However, we did not use them since the detail positions and energies are not available (Kachelrieß \& Semikoz 2006, and references therein).

Auger is located in Argentina and began to collect data from 2004 January 1. It has two systems, one to measure fluorescence in the atmosphere and the other to detect Cerenkov light from relativistic particles. The angular resolution of Auger is about $0^{\circ} 9$ (Ave 2007). Eighty-one events with $E>40 \mathrm{EeV}$ have been recorded by Auger from 2004 January 1 to 2007 August 31 but only 27 events with energies above $57 \mathrm{EeV}$ were published. Recently, another 31 new events $(E>57$ $\mathrm{EeV}$ ) were detected (The Pierre Auger Collaboration 2009); however, data are not yet available. AGASA and Yakutsk are all located in the northern hemisphere. AGASA has been operated for 12 years, and ceased operation on 2004 January 4 Its angular resolution is about 1.8 (Hayashida et al. 2000).
Up to 2000, 57 events with $E>40 \mathrm{EeV}$ have been published (Hayashida et al. 2000). Yakutsk collected 51 events with $E>40 \mathrm{EeV}$, its angular resolution is smaller than $5^{\circ}$ (Pravdin et al. 2005). Therefore, the UHECRs data used in this work include 135 events, 57 recorded by AGASA and 51 by Yakutsk in the northern hemisphere with $E>40 \mathrm{EeV}$, and 27 events recorded by Auger in the southern hemisphere with $E \geqslant 57 \mathrm{EeV}$.

Due to the different angular resolution, different energy calibration, and different sky exposure for Auger, AGASA, and Yakutsk UHECR events, we search for the possible correlations separately between the three sets of UHECRs and astrophysical objects.

\subsection{Potential Astrophysical Objects as Cosmic-Ray Sources}

The nearby AGNs used in this work are extracted from the 13th VCV AGN catalog (Veron-Cetty \& Veron 2010), with a redshift limit of $z \leqslant 0.024$ which corresponds to a GZK cutoff $\sim 100 \mathrm{Mpc}$ for the Hubble constant $H_{0}=71 \mathrm{~km} \mathrm{~s}^{-1} \mathrm{Mpc}^{-1}$. The VCV catalog includes all known AGNs reported in the literature. There are 133,336 quasars, 1374 BL Lac objects, and 34,231 active galaxies. This catalog is not complete and not uniform due to different selection criteria and telescope time devoted to different sky areas. On the other hand, the difficulties in the classification of galaxies with weak AGNlike activity result in the confusion in identification of such sources. Thus, there are some flaws when using some given particular AGN catalog to study their possible correlations with UHECRs (see Moskalenko et al. 2009). AGNs are favored as the most probable sources for accelerating particles to the extreme energies (Hillas 1984) and almost all known AGNs are listed in the catalog. In this work, we work on the VCV nearby AGNs and compare the results with that by The Pierre Auger Collaboration (2007). For the VCV AGN catalog, the incompleteness is particularly serious around the galactic plane of $|b| \leqslant 10^{\circ}$. Therefore, only the 830 nearby AGNs with $|b|>10^{\circ}$ are used in this work.

Another type of UHECR source candidates investigated in this work are Fermi sources. The Fermi/LAT Collaboration. (2009) presented the initial 3 month results for the 205 most significant $\gamma$-ray sources with energies above $100 \mathrm{MeV}$. Recently, after one-year observations, Fermi/LAT released the catalog for the all-sky $1451 \gamma$-ray sources (Abdo et al. 2010), which contains 820 identified sources and 631 unidentified sources. The Fermi/LAT first-year $\gamma$-ray source catalog (1FGL) is a complete $\gamma$-ray source sample with a $\gamma$ ray flux threshold of $>4 \times 10^{-10} \mathrm{~cm}^{-2} \mathrm{~s}^{-1}$ in the energy range $1-100 \mathrm{GeV}$. Abdo et al. (2010) identified many Fermi AGNs with redshift data. Due to the GZK cutoff, we restrict the Fermi sources with $z \leqslant 0.024$. There are only eight AGNs that satisfy this criterion. In this work, we adopt these eight objects and other Fermi sources without redshift data to search for their possible correlation with UHECRs. Because the diffuse emission dominates at low Galactic latitude $\left(|b| \leqslant 10^{\circ}\right.$ ), the Fermi sources of $|b| \leqslant 10^{\circ}$ are also discarded. In total, 635 Fermi sources are used in this work, including 262 identified sources (235 AGNs) and 373 unidentified sources.

\section{THE GALACTIC MAGNETIC FIELDS AND DEFLECTION OF UHECRS}

The GMFs have large-scale regular and small-scale turbulent components. The deflection angles of UHECRs caused by the turbulent fields are typically 1 order of magnitude 
smaller than that by the regular fields (Tinyakov \& Tkachev 2005); therefore, we ignore the turbulent component in this work. The Galactic magnetic fields in general are described as the regular magnetic field in the disk and the possible large-scale field in the halo. Previous magnetic field models for the disk are either axissymmetric (e.g. Stanev 1997) or bi-symmetric (e.g. Harari et al. 1999; Tinyakov \& Tkachev 2002). However, none of these simple models agrees well with the observations (Men et al. 2008; Sun et al. 2008).

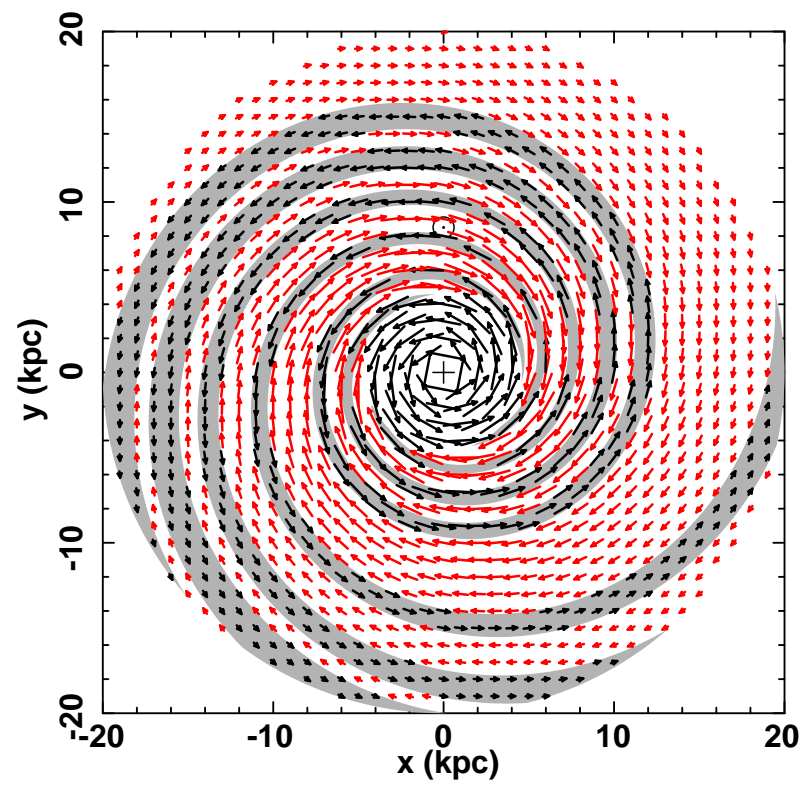

FIG. 1.- Configuration of the disk magnetic field. The shaded area outlines spiral arms given by Hou et al. (2009).

Here we developed a toy model based on rotation measures (RMs) of pulsars by Han et al. (2006). Magnetic fields in the disk are reversed from arms to inter-arms which has recently been verified by Nota \& Katgert (2010). The radial profile of the field strength can be described as $B(R)=B_{0} \exp (-(R-$ $\left.R_{0}\right) / R_{B}$ ), where $B_{0}=2.1 \mu \mathrm{G}$ is the local field strength, $R$ is the distance from the Galactic center, $R_{0}=8.5 \mathrm{kpc}$ is the galactocentric distance of the Sun, and the scale radius is $R_{B}=8.5 \mathrm{kpc}$. Here, we use the four-arm model of Hou et al. (2009) to describe the spiral structure of our Milky Way. In the polar coordinates, the $i$ th arm can be described as $R=$ $R_{i} \exp \left[\left(\theta-\theta_{i}\right) \tan \psi_{i}\right]$, where $R_{i}$ is the initial radius, $\theta_{i}$ is the start azimuthal angle, and $\psi_{i}$ is the pitch angle of the arm. The values of these parameters of four arms are given in Table 1 of Hou et al. (2009). To ease the model description, magnetic fields within $4.6 \mathrm{kpc}$ do not have reversals. The initial width of each arm is set to be $0.4 \mathrm{kpc}$ in our work. The pitch angle of the magnetic field is $-11^{\circ}$ as used by Han et al. (2006). The configuration of the disk magnetic fields in our toy model is displayed in Figure 1, which gives the counterclockwise field in the arms and the clockwise field in the inter-arm regions. Here, we remind that this is only a toy model and the fourarm model is not the best one to match all tracers for the spiral arms (Hou et al. 2009).

The halo magnetic fields consist of a dipole poloidal field and a toroidal field with opposite directions above and below the Galactic plane. The field configuration was derived from the antisymmetric RM sky revealed by the extragalactic radio sources (Han et al. 1997, 1999) and the vertical filaments in the Galactic center (Yusef-Zadehet al. 1984, 2004).
At present, it is not clear whether such toroidal fields extend from the solar vicinity to the Galactic center and what the scaleheight they have. Here we use the formula given by Prouza \& Smída (2003) in the Cartesian coordinates to describe the toy model:

$$
\left\{\begin{array}{l}
B_{x}=-B_{T} \sin (\phi) \operatorname{sign}(\mathrm{z}) \\
B_{y}=B_{T} \cos (\phi) \operatorname{sign}(\mathrm{z}),
\end{array}\right.
$$

where $B_{T}$ is given by

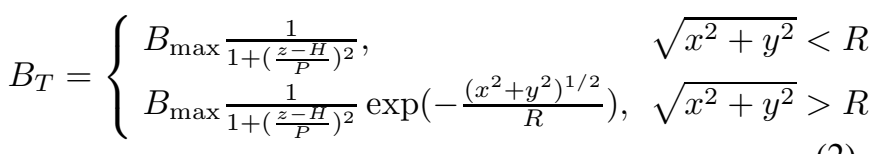

Here $R=15 \mathrm{kpc}$ is the radius of the toroidal field, $H=$ $1.5 \mathrm{kpc}$ is the scaleheight of the toroidal disk, $P=0.3 \mathrm{kpc}$ is the halfwidth of the Lorentzian distribution, and the maximal value of the magnetic field is $B_{\max }=1 \mu \mathrm{G}$.

The poloidal field is modeled as a dipole with a cylinder (height $300 \mathrm{pc}$, diameter $200 \mathrm{pc}$ ) in the Galactic center. The existence of the dipole field is questioned by Taylor et al. (2009) and Mao et al. (2010). However, their data in the Galactic pole region clearly support the local vertical field. In this work, we try to include the dipole field in our toy model. In the polar coordinates the poloidal field strength is (in the $x z$-plane, $\theta$ ranges from 0 to $\pi$ and goes from north to south pole)

$$
B=B_{P} \sqrt{3 \cos ^{2}(\theta)+1} .
$$

Then the Cartesian components of the poloidal field are

$$
\left\{\begin{array}{l}
B_{x}=1.5 B_{P} \sin 2 \theta \cos \phi \\
B_{y}=1.5 B_{P} \sin 2 \theta \sin \phi \\
B_{z}=B_{P}\left(3 \cos ^{2} \theta-1\right)
\end{array}\right.
$$

where

$$
B_{P}= \begin{cases}100 / R_{P}^{3} & 5<R_{P}<15 \\ 1 & 2<R_{P}<5 \\ 0.2 / R_{P}^{3} & 0.1<R_{P}<2 \\ 2000 & R_{P}<0.1 \text { and }|z|<0.15\end{cases}
$$

Here $R_{P}=\sqrt{x^{2}+z^{2}} . R_{P}$ and $z$ are in units of kpc, and $B_{P}$ is in units of $\mu \mathrm{G}$. The constants in $B_{P}$ were selected to meet the characters of observed filaments in the Galactic center (1$2 \mathrm{mG}$; Morris \& Serabyn 1996), and a $0.2 \mu \mathrm{G}$ vertical field component in the vicinity of the Sun (Han \& Qiao 1994).

In our toy model, the field transition from the arms to the inter-arms is not smooth, the influence of the bar in the Galactic center is not considered yet. The detailed model is beyond the scope of this paper. We will investigate how much UHECRs are reflected by each magnetic field component in the model.

The cosmic rays are deflected in the GMFs because of the Lorentz force. Following Kachelrieß et al. (2007), the net deflection can be approximated as

$$
\Theta \approx \frac{Z q_{e}}{p c} \int d \vec{l} \times \vec{B}_{t}
$$

where $Z q_{e}$ is the charge of cosmic-ray particles, $p$ is the momentum along the line of sight (LOS), and $\vec{B}_{t}$ is the field component perpendicular to the LOS. The integral is along the LOS from the source to the observer. In our work, the 

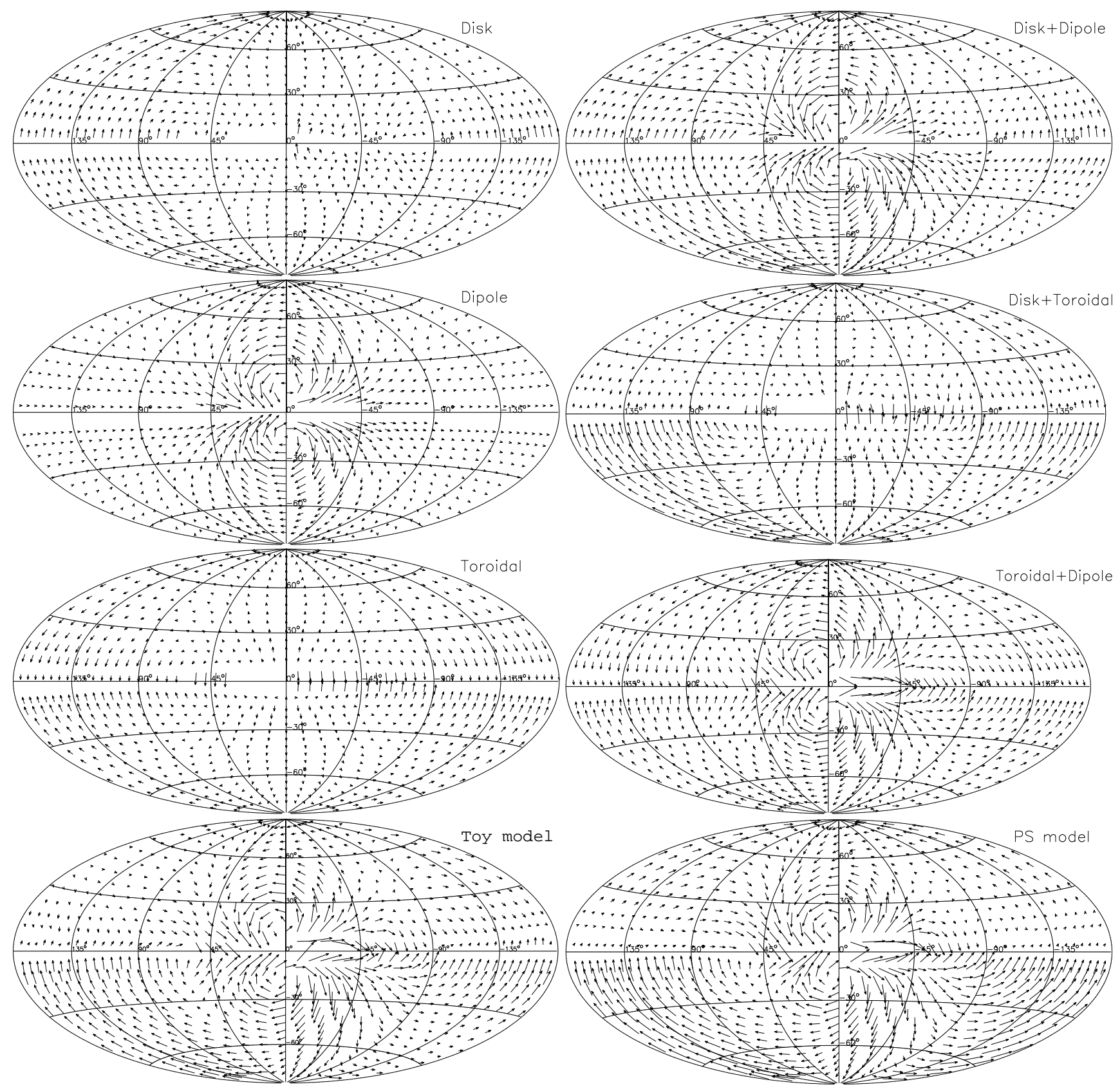

FIG. 2.- Deflection maps of CR protons of various GMF components and their combinations in our toy model and the PS model (Prouza \& Smída 2003). The energy of CRs is fixed to be $40 \mathrm{EeV}$. All the maps are plotted in the Galactic coordinates. Each arrow goes from the observed direction of a CR on the Earth to the actual incoming direction of the source.

Hammurabi code (Waelkens et al. 2009) is used to calculate the all-sky deflections.

In Figure 2, we show the deflection angle maps of UHECRs for various magnetic field components and their combinations in the toy model, and also the PS model (Prouza \& Śmída 2003) which has a different disk component. The energy of $\mathrm{CR}$ protons is fixed to be $40 \mathrm{EeV}$. The disk field generates a strong deflection near the Galactic disk and in the high latitude. The toroidal field is similar to the disk field and has a large deflection near the disk, while the orientations are opposite above and below the disk. The dipole component has very strong deflections in the Galactic center region. Deflection maps by our toy model and the PS model have some similar features: (1) the maximal deflections take place in the inner Galactic disk and Galactic central regions; (2) in the southern sky of the outer Galaxy, the deflection is as strong as that in the Galactic center, while in the northern sky, a very small deflection occurs. This is a result of combining the toroidal and the dipole magnetic field components. However, there is quite difference in some regions between these two models: in the northern sky, our model generates smaller deflections from $l=0^{\circ}$ to $l=180^{\circ}$, and the orientation is contrary to the PS model in the region of $-90^{\circ}<l<-180^{\circ}$; in the southern sky, the PS model has larger deflections, particularly in the region of $-90^{\circ}<l<-180^{\circ}$.

The energy of observed UHECRs in our data sets varies from $40 \mathrm{EeV}$ to more than $200 \mathrm{EeV}$. The deflections for the de- 


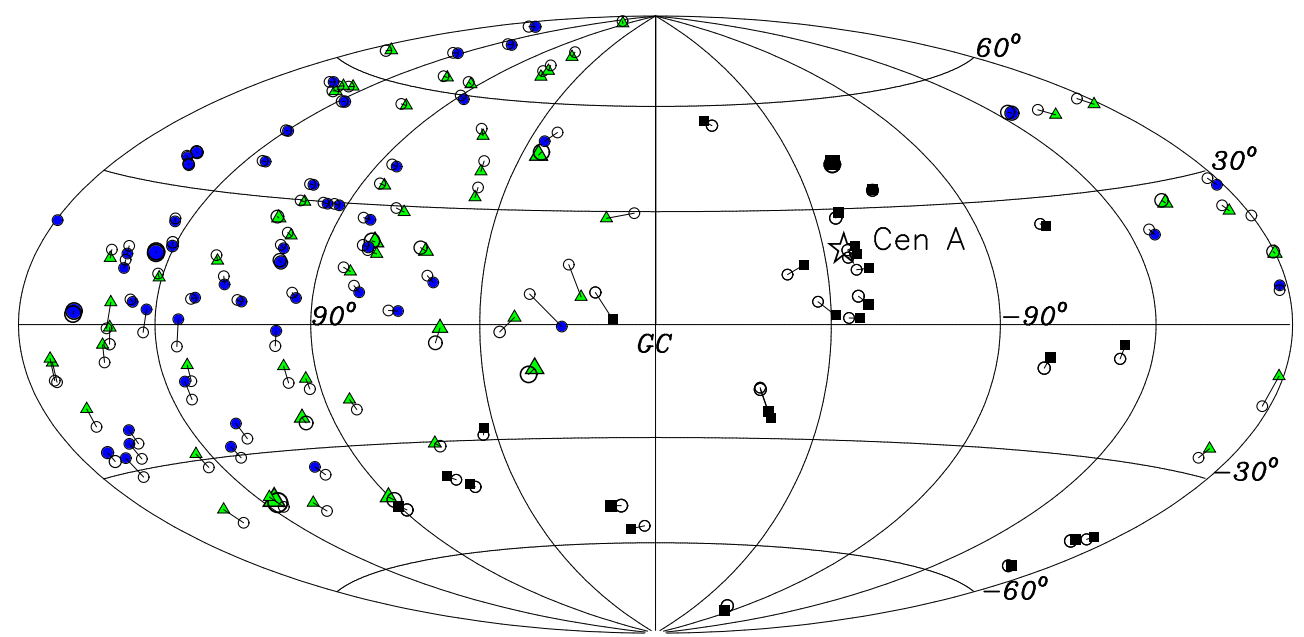

FIG. 3.- Deflection of 135 detected UHECRs according to our GMF toy model (in the Galactic coordinates). The circles are the original arrival directions of 135 UHECRs observed on the Earth, the squares, triangles, and dots are the deflection corrected positions of the Auger, AGASA, and Yakutsk CR events, respectively. The size of symbols is proportional to the CR energy. The star marks the Cen A.

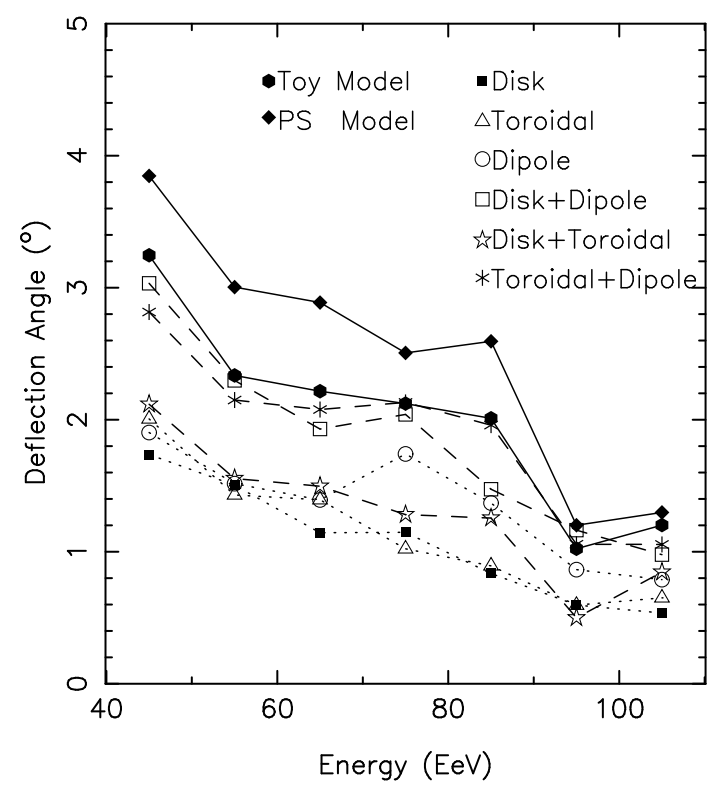

FIG. 4.- Average deflection angles of 135 detected UHECRs as a function of energy by our GMF toy model and the PS model (Prouza \& Smída 2003). The solid lines are our model (black dot) and PS model (black diamond), dotted lines are three GMF components, dashed lines are their combinations.

tected UHECR events according to our toy model are shown in Figure 3 Figure 4 shows the average deflection angles of 135 UHECRs by the two GMF models and the various magnetic component combinations. We found that the deflections decrease with the CR energy as expected. Magnetic fields in our model generate smaller deflections than those in the PS model. CR events with energy below $90 \mathrm{EeV}$ are deflected by an angle $\lesssim 3^{\circ}$. The arrival directions of the events with energy above $90 \mathrm{EeV}$ are deflected less than $2^{\circ}$. The deflection angles are generally similar to the angular resolution of the UHECR detectors $\left(\sim 1^{\circ}-2^{\circ}\right)$; therefore the GMF deflection correction is still desired in understanding the origin of UHECRs.

\section{CORRELATIONS BETWEEN THE UHECR EVENTS AND POTENTIAL COSMIC-RAY SOURCES}

In this work, we try to correct the deflections of the UHECR events by the GMFs, and then search for possible correlations between three UHECR data sets (Auger, AGASA, and Yakutsk) and nearby AGNs and Fermi sources. We will compare the correlation results with and without the deflection correction. Here, we first introduce the correlation analysis method.

\subsection{The Correlation Analysis Method}

To carry out the correlation analysis, we use the angular correlation function method described in Tinvakov \& Tkachev (2001a.b), Gorbunov et al. (2002, 2004), and Gorbunov \& Troitsky (2005). For a sample with $n_{r}$ cosmic-ray events, we count the number $N_{c s}$ for UHECR-source pairs within a given angle $\delta$, which is called "bin size" and various from 0 to a large angle. We count 1 if at least one potential cosmic-ray source (such as AGNs) falls into "the bin" and count 0 if no source falls into the bin. To check the chance probability, we use Monte Carlo simulations. We generate a large number (e.g., with $N=10^{4}$, $10^{6}$ for some cases) of simulated sets of UHECR events, each set has the same number of events as the real sample. The simulated UHECRs are isotropic and the locations of simulated events are random. The distribution of locations is constrained by the overall exposure of the UHECR detectors. For a given $\delta$, we first count the number of UHECR-source pairs $N_{m c}$ for each simulated UHECR sample, and obtain a mean $\bar{N}_{m c}$ and the variance $\sigma_{m c}$ from $N$ simulation sets. The exposure of Auger, AGASA, and Yakutsk depends on the celestial declination (The Pierre Auger Collaboration 2008; Takeda et al. 1999; Ivanov et al. 2003b). The Auger exposure used in our work is obtained from the fitting of the declination distribution of low-energy Auger events $\left(E<10 \mathrm{EeV}\right.$, from the Auger Web site $\left.{ }^{3}\right)$ with a third-order polynomial function. The AGASA exposure function is taken from Takeda et al. (1999). The Yakutsk exposure function is derived from Ivanov et al. (2003b). The probability that the observed UHECR-source pairs are in coincidence with a random distribution is estimated by

\footnotetext{
${ }^{3} \mathrm{http} / / /$ auger.colostate.edu/ED/
} 
TABLE 1

Correlation Between UHECRs With AGNs And Fermi $\gamma$-RAy Sources With or Without Deflection CorRections by GMFs

\begin{tabular}{|c|c|c|c|c|c|c|c|c|c|c|c|c|c|c|c|c|}
\hline \multirow{3}{*}{$\begin{array}{c}(1) \\
\text { Sources }\end{array}$} & \multirow{3}{*}{$\begin{array}{l}\text { (2) } \\
\text { GMF }\end{array}$} & \multicolumn{4}{|c|}{ Auger Events } & \multicolumn{5}{|c|}{ AGASA Events } & \multicolumn{6}{|c|}{ Yakutsk Events } \\
\hline & & (3) & & (5) & (6) & (7) & & & 7 & (11) & (12) & 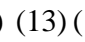 & ( & (15) & (16) & (17) \\
\hline & & $\delta\left(^{\circ}\right)$ & & $\bar{N}_{m c}$ & $P$ & $\sigma$ & & & $\bar{N}_{m}$ & $P$ & $\sigma$ & & & $\bar{N}_{m c}$ & $P$ & \\
\hline \multirow{9}{*}{ VCV/AGN } & Non & 2.4 & 17 & 6.7 & $1 \times 10^{-6}$ & 4.7 & 1.4 & 11 & 6.7 & 0.066 & 1.8 & 3.0 & 19 & 17.9 & 0.420 & .4 \\
\hline & Toy model & 3.6 & 20 & 10.5 & $2 \times 10^{-5}$ & 4.1 & 2.1 & 17 & 11.5 & 0.049 & 1.9 & 3.6 & 25 & 21.4 & 155 & .2 \\
\hline & Disk & 3.3 & 21 & 9.5 & $<1 \times 10^{-6}$ & 5.0 & 0.6 & 4 & 1.4 & 0.048 & 2.3 & 0.3 & 1 & 0.3 & .277 & .2 \\
\hline & Dipole & 2.1 & 14 & 5.5 & $1.6 \times 10^{-5}$ & 4.2 & 2.7 & 22 & 18.3 & 0.172 & 1.1 & 3.3 & 22 & 19.9 & .307 & 0.7 \\
\hline & Toroidal & 3.3 & 20 & 9.5 & $<1 \times 10^{-6}$ & 4.6 & 3.6 & 29 & 25.1 & .164 & 1.1 & 3.0 & 20 & 17.4 & 237 & .9 \\
\hline & Disk+Dip. & 3.0 & 18 & 8.5 & $2 \times 10^{-5}$ & 4.2 & 1.5 & 13 & 7.1 & 0.019 & 2.4 & 2.4 & 15 & 13.4 & 350 & 0.5 \\
\hline & Disk+Toro. & 3.9 & 21 & 11.4 & $2 \times 10^{-5}$ & 4.2 & 2.4 & 18 & 14.6 & 0.179 & 1.1 & 3.0 & 21 & 17.4 & 0.148 & 1.2 \\
\hline & Tor & 3.3 & 19 & 9.5 & $2 \times 10^{-5}$ & 4.2 & 2.4 & 15 & 14.6 & 0.499 & 0.1 & 3.6 & 25 & 21.4 & 155 & 1.2 \\
\hline & PS ${ }_{1}$ & 3.0 & 18 & 8.5 & $2 \times 10^{-5}$ & 4.2 & 1.5 & 11 & 6.7 & .062 & 1.8 & 1.2 & 7 & 2 & 17 & .5 \\
\hline \multirow[t]{9}{*}{ 1FGL/All } & Non & 4.0 & 17 & 13.5 & 0.091 & 1.5 & 3.0 & 25 & 20.9 & 0.153 & 1.2 & 2.6 & 16 & 11.7 & .096 & 1.5 \\
\hline & Toy model & 0.9 & 5 & 1.0 & $2.5 \times 10^{-3}$ & 4.1 & 2.4 & 18 & 12.9 & 0.070 & 1.7 & 4.2 & 28 & 22.7 & .053 & 1.8 \\
\hline & Disk & 2.4 & 10 & 6.1 & 0.056 & 1.9 & 2.7 & 22 & 17.1 & 0.095 & 1.5 & 4.2 & 29 & 23.9 & & 17 \\
\hline & $\mathrm{Di}$ & 4.2 & 19 & 14.3 & 0.0 & 2.1 & 5.7 & 45 & & 0.1 & 1.1 & 4.2 & 29 & & & .9 \\
\hline & & 0.6 & 2 & 0.4 & $0.0^{\circ}$ & 2.4 & 4.8 & 41 & 35.2 & 0.038 & 1.9 & 5.1 & 32 & 27.7 & 80 & .6 \\
\hline & Disl & 1.8 & 9 & 3.7 & $5.3 \times 10^{-3}$ & 3.0 & 1.8 & 14 & 8.4 & & 2.1 & 3.6 & 25 & 19.7 & 0.066 & 1.7 \\
\hline & Disk & 1.8 & 7 & 3.7 & 0.064 & 1.9 & 3.6 & 31 & 24.9 & 0.056 & 1.7 & 5.1 & 33 & 27.7 & 0.034 & 2.0 \\
\hline & & 3.6 & 17 & 11.3 & $9.1 \times 10^{-3}$ & 2.5 & 5.4 & 42 & 38.0 & 0.106 & 1.4 & 3.9 & 27 & 20.7 & 0.028 & .1 \\
\hline & PS & 2.1 & 12 & 4.9 & $6.0 \times 10^{-4}$ & 3.7 & 1.5 & 11 & $\sigma_{-1}$ & 0.019 & 2.4 & 3.3 & 23 & 1 & 42 & .9 \\
\hline \multicolumn{2}{|c|}{ 1FGL/AGN Non } & 7.6 & 20 & 14.7 & 0.013 & 2.4 & 3.8 & 23 & 15.8 & 0.025 & 2.2 & 5.2 & 21 & 19.8 & 0.416 & 0.4 \\
\hline & Toy $n$ & 0.9 & 2 & 0.3 & 0.037 & 3.1 & 2.7 & 15 & 7.8 & $7.0 \times 10^{-}$ & 2.8 & 3.6 & 15 & 11.6 & 0.150 & 1.2 \\
\hline & Di & 8.1 & 20 & 15.1 & 0. & 2.4 & 2.7 & 15 & 8.6 & & 2.4 & 0.6 & 1 & & & 0.9 \\
\hline & & 3.3 & 9 & 4.0 & $9.3 \times 10^{-3}$ & 2.8 & 4.5 & 27 & 20.0 & & 2.0 & 4.2 & 17 & 1 & 3 & \\
\hline & Tor & 7.5 & 19 & 13.8 & 0.012 & 2.4 & 4.2 & 20 & 17.4 & 0.255 & 0.8 & 3.6 & 12 & 11.6 & 498 & .2 \\
\hline & Disk & 1.8 & 4 & 1.2 & 0.03 & 2.7 & 1.8 & 10 & 4.0 & $5.2 \times 10^{-}$ & 3.2 & 3.6 & 16 & 12.2 & 0.124 & .3 \\
\hline & Disk+Toro. & 8.1 & 20 & 15.1 & 0.010 & 2.4 & 3.6 & 20 & 13.4 & 0.027 & 2.1 & 3.9 & 15 & 13.1 & .305 & 0.7 \\
\hline & & 3.9 & 9 & 5.1 & 0.0 & 2.0 & 1.8 & 6 & 3.9 & 0. & 1.1 & 3.9 & 15 & 13.1 & 0.305 & 0.7 \\
\hline & & 5.4 & 13 & .7 & & 1.9 & 1.5 & 9 & 2.6 & $7.0 \times 10^{-}$ & 4.1 & 3.3 & 12 & 10.3 & 12 & \\
\hline \multicolumn{2}{|c|}{ 1FGL/Un-idNo } & 7.0 & 21 & 18.3 & 0.1 & 1.5 & 6.0 & 36 & 32.6 & 0.201 & 1.0 & 2.6 & 10 & 5.5 & 38 & 2.1 \\
\hline & Toy $n$ & 3.0 & 11 & 6.1 & 0.021 & 2.4 & 5.4 & 29 & 25.6 & 96 & 1.0 & 6.6 & 30 & 23.3 & 0.015 & 2.2 \\
\hline & Dis & 6.0 & 19 & 15.3 & 0.05 & 1.8 & 5.4 & 32 & 27.8 & 0.151 & 1.2 & 5.1 & 23 & 17.4 & 0.052 & .8 \\
\hline & & 1.2 & 4 & 1.2 & & 2.6 & 6.0 & 37 & 31 & & 1.5 & 6.9 & 30 & & & 6 \\
\hline & & 8.1 & 21 & 19.0 & & 1.5 & 6.3 & 35 & 32 & & 0.8 & 5.1 & 23 & & 0.029 & \\
\hline & & 0.9 & 3 & 0.7 & 0.0 & 2.9 & 6.3 & 38 & 32.2 & & 1.7 & 6.0 & 28 & 21.6 & 0.029 & 2.0 \\
\hline & Disk+Toro. & 7.8 & 21 & 18.6 & 0.085 & 1.6 & 6.0 & 32 & 30.0 & 0.335 & 0.6 & 5.7 & 28 & 19.32 & $2.9 \times 10^{-3}$ & 2.8 \\
\hline & Torc & 3.6 & 12 & 8.1 & 0.066 & 1.7 & 5.1 & 29 & 24.9 & 0.151 & 1.2 & 7.5 & 32 & 26.8 & 0.041 & 1.9 \\
\hline & PS model & 1.8 & 8 & 2.5 & $7 \times 10^{-}$ & 3.7 & 0.6 & 1 & 0.5 & 0.387 & 0.7 & 6.6 & 30 & 23.9 & 0.028 & .0 \\
\hline
\end{tabular}

$$
P(\delta)=\frac{\text { number of simulated sets with } N_{m c} \geqslant N_{c s}}{N} .
$$

The significance of the correlation can be defined as $\sigma(\delta)=$ $\frac{N_{c s}-\bar{N}_{m c}}{\sigma_{m c}}$. We emphasize that $P$ and $\sigma$ vary with $\delta$. Larger chance probability $P(\delta)$ indicates that the observed pairs of cosmic rays and astrophysical objects are more likely the statistical coincidence of random isotropic UHECR events. The higher significance corresponds to a smaller $P(\delta)$ which suggests that the objects of the pairs are more likely to be potential UHECR sources. In this paper, $P<10^{-2}$ is believed as an indicator of some correlation. We use this method to evaluate the possible correlations between the Auger/AGASA/Yakutsk events and the possible cosmic-ray sources.

\subsection{The Correlation Between UHECRs and VCV AGNs}

All sky distribution of AGNs in our selected sample and the deflection-corrected positions of the 135 UHECR events are presented in Figure 5. We perform the correlation analysis separately for Auger, AGASA, and Yakutsk events. The correlation results for the UHECRs with and without deflection corrections are presented in Table 1 . We also present the correlation results for deflection corrections using the PS model (Prouza \& Śmída 2003) and various GMF components and their combinations in our toy model. In Figures 6 and 7 we show the results of pair counting and probability analysis for the deflection-corrected UHECRs by our GMF toy model.

In Figures 6, 7, and Table 1, we found that the number of Auger UHECR-AGN pairs is more than that of simulated isotropic random UHECR samples, which suggests that the Auger UHECRs are anisotropic and some- 


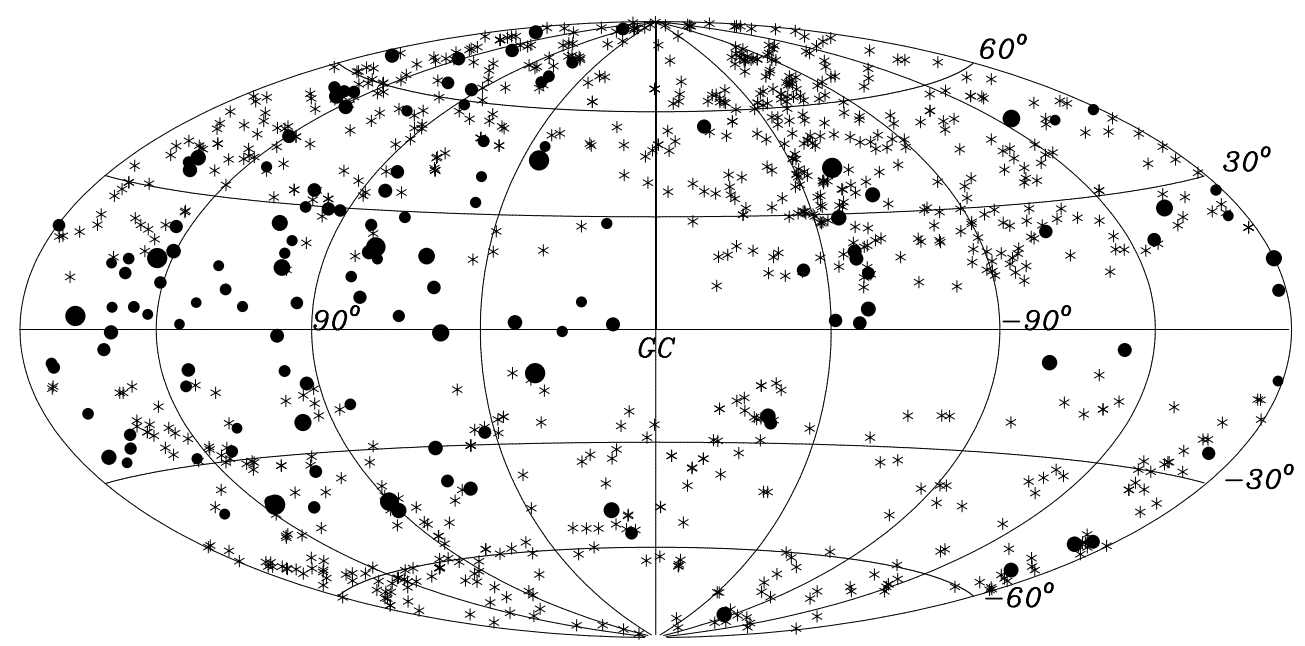

FIG. 5.- Sky map for 830 nearby VCV AGNs (*) and 135 UHECRs (dots) in the Galactic coordinates with the deflection corrected by our GMF toy model. Size of black dots are proportional to the cosmic-ray energy.

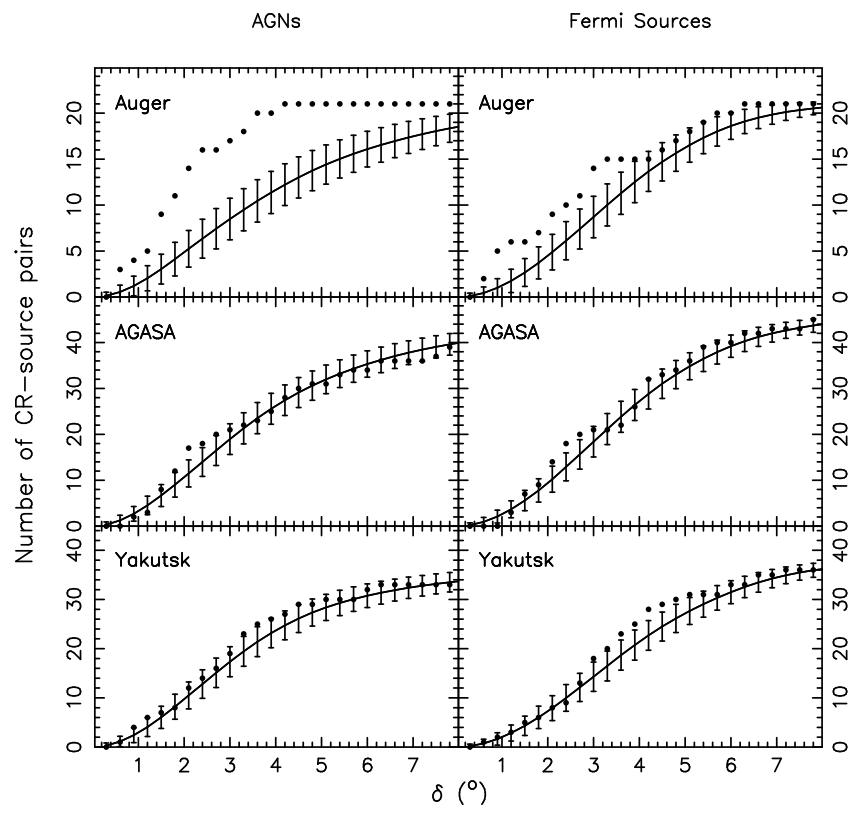

FIG. 6.- Number of UHECR-source pairs (dot) for nearby VCV AGNs (left column) and Fermi sources (right column), as a function of the angular separation (bin size) $\delta$. The solid line is the Monte-Carlo simulated average number of UHECR-source pairs with errorbar of $\pm 1 \sigma$, derived from random isotropic distribution of simulated cosmic-ray events.

how correlated with VCV AGNs, supporting the results of The Pierre Auger Collaboration (2007, 2008). For the deflection-corrected Auger UHECRs by our GMF model and the PS model, the chance probability is $2 \times 10^{-5}$, and the correlation significance is about $4 \sigma$, which is similar to but slightly less significant than the results of a chance probability of $1 \times 10^{-6}$ and correlation significance of $4.7 \sigma$ without deflection correction. In Table 1 , we found that the similar correlation results are also presented in the cases of various GMF components and the PS model, which indicate that some of UHECRs probably come from a few of AGNs. The marginal correlation significance of a few sigma $(\lesssim 5 \sigma)$ only suggests that most UHECRs do not have AGN counterparts. To further evaluate the deflec-

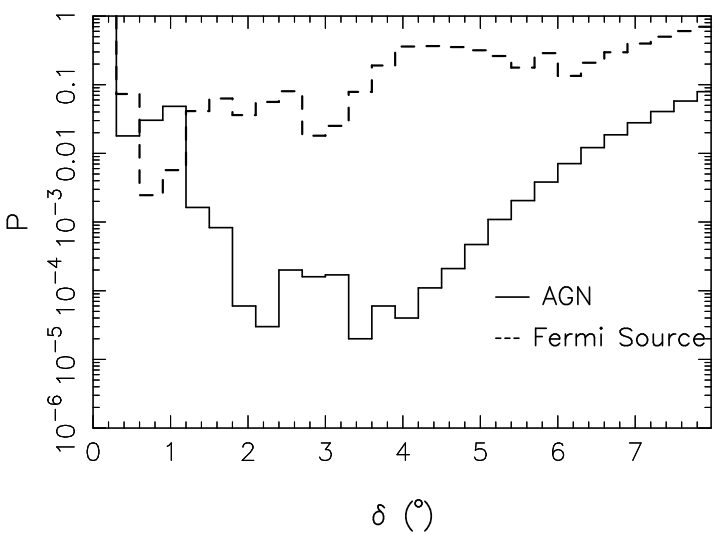

FIG. 7.- Chance probability $P(\delta)$ as a function of the angular separation $\delta$ of Auger UHECR events from the VCV AGNs and the Fermi sources (dashed line), for example.

tion effect, we use the deflection-corrected Auger events to search for match pairs, and compare them with the results of The Pierre Auger Collaboration (2008) who did not consider the deflections. Using the 442 nearby VCV AGNs from 12 th edition, we found 15 UHECR-AGN pairs, instead of 20 pairs reported by The Pierre Auger Collaboration (2008) with the parameters $z_{\max }=0.017, \psi=3^{\circ} 2$, and $E_{\mathrm{th}}=57 \mathrm{EeV}$. Note that the fewer pairs imply the weaker correlation between UHECRs and AGNs after deflection correction. Some of the matched pairs reported by The Pierre Auger Collaboration (2008) are probably due to random match. The Auger UHECR-AGN correlation is also weakened when the update Auger UHECR data are used (The Pierre Auger Collaboration 2009).

As shown in Figures 6, 7, and Table 1, we found no correlation between the AGASA and Yakutsk events and AGNs.

\subsection{Correlation Between UHECRs and Fermi $\gamma$-ray Sources}

High energy $\gamma$-ray emissions are thought to be a distinctive feature of the possible source of UHECRs (Gorbunov et al. 2002). Torres et al. (2003) searched for possible correlation between the third EGRET sources and AGASA UHECR events, no correlation was found. The GeV AGNs detected 


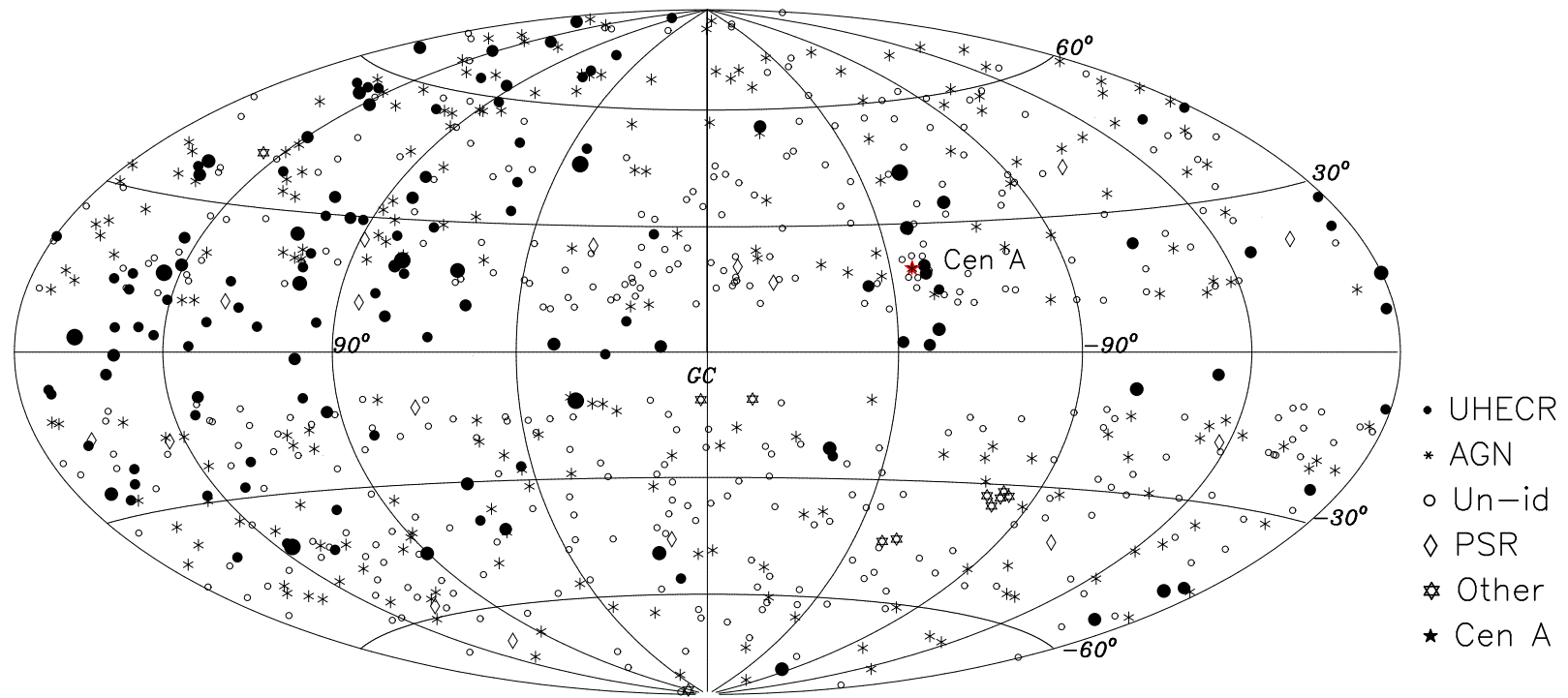

FIG. 8. - All sky maps of 135 UHECR events and 635 Fermi sources in Galactic coordinates with the deflection corrected by our GMF toy model. Black dots are the locations of 135 UHECRs weighted with their energy.

by the Fermi/LAT should be most energetic AGNs which may have powerful ability to accelerate the charged particles to ultrahigh energy bands. Most important is that the selected GeV AGNs in the Fermi catalog are relatively complete above the $\gamma$-ray flux threshold. We therefore investigate the possible correlations between Fermi sources and the Auger/AGASA/Yakutsk UHECR events considering the deflection of the GMFs. At the final stage of this work, we noticed that Mirabal \& Oya (2010) did the similar work as us and studied the possible correlation between the Fermi/LAT First Source Catalog with the public Auger UHECRs. They found that 12 of the 27 Auger UHECRs arrived within $3^{\circ} .1$ of Fermi sources, similar to the matches of artificially random distribution of UHECR samples. They concluded for no correlation. However, the possible correlation of UHECRs and Fermi sources is needed to be re-examined after the deflections by the GMFs and the GZK effect are considered.

The Fermi/LAT 1FGL catalog contains 1451 point sources, including 820 identified objects: 62 pulsars, 295 BL Lacs, 278 FSRQs, 120 other types of AGNs, and 65 other $\gamma$-ray sources. We remove the Fermi sources with redshift $z>0.024$ by considering the GZK cutoff, and also neglect the sources located in the Galactic disk with $|b| \leqslant 10^{\circ}$. Figure 8 presents the sky distributions of the remaining 635 Fermi/LAT $\gamma$-ray sources and the deflection-corrected UHECR events.

The correlation results for the Fermi $\gamma$-ray sources and UHECRs are listed in Table 1, In the right panel of Figure 6, we also present the number of UHECR-source pairs as a function of the bin size $\delta$. In the case of deflection-corrected Auger events by our GMF model, a small excess appears around $\delta \sim 0^{\circ} 9$, with a chance probability of $P \sim 2.5 \times 10^{-3}$, and a significance level of $\sim 4.1 \sigma$. A correlation with a chance probability of $P \sim 6.0 \times 10^{-4}$, and a significance level of $\sim 3.7 \sigma$ is also found for the case with deflection correction by the PS model. These correlations with marginal significance probably indicate that a few Fermi sources are related to the UHECRs. However, most of the Fermi sources are not potential sources of UHECRs. No evidence of correlation is found in the case of the observed positions of Auger events. In the cases of the AGASA and Yakutsk data, no sig- nificant correlations are found, though many UHECRs have a Fermi source within $3^{\circ}$ from their arrival positions. In order to evaluate the effect of deflections on the correlation analysis and compare our results with those of Mirabal \& Oya (2010), we also try to use all 1451 sources in the 1FGL for correlation with Auger UHECRs. We found that the number of matched UHECR-source pairs can be reproduced by the simulated isotropic UHECR samples for the UHECR sample without deflection correction, which is consistent with the conclusion of no correlation by Mirabal \& Oya (2010). When we exclude the sources with $|b| \leqslant 10^{\circ}$ and consider the deflection correction by our GMF model, a marginal correlation is found with a chance probability of $P \sim 9.8 \times 10^{-3}$ and a significance level of $\sim 3.1 \sigma$.

Fermi sources contain several types of objects, such as pulsars, AGNs, and unidentified sources. It is interesting to see if possible correlations exist between some types of Fermi sources and UHECRs. The correlation analysis results for the Fermi AGNs and UHECRs are presented in Table 1. The Fermi AGNs are weakly correlated with AGASA UHECRs after the deflection corrected by our GMF model and the PS model. Three hundred seventy-three unidentified Fermi sources are also weakly correlated with Auger UHECRs after the deflection corrected by the PS model. The correlation results for UHECRs with deflection corrections using various GMF components and their combinations in our toy model are also presented in Table 1

Fermi 1FGL catalog has 8 objects of redshift $z \leqslant 0.024$ : NGC $253(z=0.001)$, NGC $4945(z=0.002)$, Centaurus A (Cen A, $z=0.002)$, M87 $(z=0.004)$, ESO 323-G77 $(z=0.015)$, NGC 6951 $(z=0.005), \operatorname{NGC} 1275(z=0.018)$, and M-82 $(z=0.001)$. We found that 4 of these 8 objects have UHECR counterpart(s) within $3{ }^{\circ} 1$ from them, after the deflections are corrected by our toy model. Mirabal \& Oya (2010) found 2 Fermi objects (NGC 4945 and Cen A) within $3^{\circ} 1$ from 3 of the 27 Auger UHECRs. When we consider the deflection correction, we find one Auger UHECR event within 3.1 ${ }^{\circ}$ from NGC 4945, Cen A and ESO 323-G77. NGC 6951 also has one Yakutsk event within $3^{\circ} 1$.

The Cen A is the nearest FR II radio galaxy (Israel 
1998), which has been long proposed as a possible source of UHECRs (Cavallo 1978; Romero 1996). Cen A was detected at $\mathrm{MeV}$ to $\mathrm{GeV}$ energies by the Fermi/LAT (The Fermi/LAT Collaboration. 2009). The Pierre Auger Collaboration (2007, 2008) pointed that 4 of the 27 events were possibly associated with Cen A (e.g. Moskalenko et al. 2009; Kachelrieß et al. 2009). From Figure 3, we found that the arrival directions of cosmic ray in the region of Cen A are not significantly corrected by the GMF model. Two of UHECR events in the 27 published Auger data set are very close to Cen A. Considering heavier composition of UHECRs, Piran (2010) suggested that Cen A is the only active potential source of heavy nuclei UHECRs within a few Mpc for the GZK cutoff. The heavy nuclei suffer a larger deflection which can erase any correlation with their source. If all detected UHECRs are produced by merely Cen A, which come to our Galaxy and suffer different deflection via different paths and finally arrive at the Earth from various directions, they should show some kind of concentration around the source direction for many lighter nuclei, depending on the detailed composition and magnetic deflection. In Figure 8 , there is some indication for such a concentration within about $20^{\circ}$ near Cen A. While, other UHECRs coming from other very different directions may have other accelerating sources rather than Cen A.

NGC 4945 is identified as a Seyfert galaxy (Véron-Cetty \& Véron 2006), also known as a starburst galaxy (Lenc \& Tingay 2009). Lenc \& Tingay (2009) identified a non-thermal source with a jet-like morphology near the AGN of NGC 4945. ESO 323-G77 is identified as a Seyfert galaxy having strong $\mathrm{Fe}_{I} I$ emission (Fairall 1986), however, no compact radio core or a radio excess has been detected (Corbett et al.2003). NGC 6951 is known as a LINER galaxy and a bipolar outflow which seems to be associated with a nuclear jet has been reported by Storchi-Bergmann et al. (2007). However, it is not clear whether the possible radio jets from NGC 4945 and NGC 6951 could be the accelerator of UHECRs.

\section{SUMMARY AND DISCUSSION}

We collected 135 published UHECR events including 57 UHECRs recorded by AGASA with energy $E>40 \mathrm{EeV}$, 51 events observed by Yakutsk, both located in the northern hemisphere, and 27 events with energy $E \geqslant 57 \mathrm{EeV}$ detected by Auger located in the southern hemisphere. We use a new GMF toy model constrained by updated measurements to evaluate the deflection effects on the arrival directions of UHECRs. Considering the possible deflection correction by our toy model and the PS model, as well as the different magnetic field components in our model, we search for the possible correlations of UHECRs with nearby AGNs extracted from the new 13th VCV AGN catalog of Veron-Cetty \& Veron (2010) and the Fermi/LAT First Source Catalog of $\gamma$-ray sources. We found a correlation between the Auger UHECR events and nearby VCV AGNs with a chance probability of $2 \times 10^{-5}$, and a significance level of $\sim 4 \sigma$. Using the same data as The Pierre Auger Collaboration (2008), we found fewer UHECR-AGN pairs when deflection is considered, which implies the weakened correlation. A marginal correlation was found between the Auger events and the first year Fermi $\gamma$-ray sources with a significance level of $\sim 4 \sigma$ if the deflection by the GMF model is considered. Some Fermi sources of nearby AGNs, NGC 4945, ESO 323-G77, NGC 6951, and Cen A, may be related to UHECRs within $3^{\circ} 1$. For AGASA and Yakutsk UHECRs, no evidence of significant correlation is found for the nearby AGNs or the Fermi sources because the matched pairs can be reproduced by the simulated random isotropic UHECR samples, though some $\gamma$-ray point sources are coincident with the UHECR events within $2^{\circ}$.

The correlations of UHECRs with some astrophysical objects suggest that at least some of the UHECRs are protons (The Pierre Auger Collaboration 2007, 2008). However, most UHECRs seem to come from various directions and do not associate with known astrophysical objects, which indicates that the majority of UHECRs might suffer larger deflections in the trajectory, due to either the unknown extragalactic magnetic fields or the heavy nuclei component of UHECRs (Piran 2010). The deflection of heavy UHECRs by the GMF models is proportional to the charge of nuclei, which leads to a very large deflection angle, for example tens of degrees for iron, and then any correlation discussed in this work can be diminished (Gureev \& Troisky 2010). If the primaries of the UHECRs are heavy nuclei, instead of proton, the identification of UHECR sources would be very difficult. Obviously, the understanding of UHECR origin will strongly depend on our knowledge about the strength and configuration of the Galactic and extragalactic magnetic fields, which definitely needs more measurements (Han 2008).

The authors are very grateful to the referee and Professor T. Stanev for valuable comments and suggestions. We thank Dr. Chen Wang for help and Dr. Zhonglue Wen, Mr. Pengfei Wang and Tao Hong for helpful discussions. We thank Professor Chih Kang Chou, Xuyang Gao, Jun Xu, and Hui Shi for improving our English. The Fermi $\gamma$-ray point sources are available in the Web site http://fermi.gsfc.nasa.gov/ssc/data Authors are supported by the National Natural Science Foundation of China (10803009, 10821061, and 10833003) and the National Key Basic Science Foundation of China (2007 CB815403).

\section{REFERENCES}

Abbasi, R. U., et al.2004, ApJ, 610, L73

Abbasi, R. U., et al.2006, ApJ, 636, 680

Abbasi, R. U., et al. 2008, Physical Review Letters, 100, 101101

Abdo, A. A., et al. 2010, ApJ, 715, 429

Abraham, J., et al. 2004, Nuclear Instruments and Methods in Physics Research A, 523, 50

Atwood, W. B., et al.2009, ApJ, 697, 1071

Ave, M. 2007, Proc. 30th ICRC, 4, 307

Bird, D. J., et al.1994, ApJ, 424, 491

Blasi, P., Epstein, R. I., \& Olinto, A. V. 2000, ApJ, 533, L123

Cavallo, G. 1978, A\&A, 65, 415

Corbett, E. A., et al. 2003, ApJ, 583, 670
Dolag, K., Grasso, D., Springel, V., \& Tkachev, I. 2004, Sov.J.Exp. Theor. Phys. Lett., 79, 583

Dolag, K., Grasso, D., Springel, V., \& Tkachev, I. 2005, J. Cosmol. Astropart. Phys., JCAP01(2005)009

Fairall, A. P. 1986, MNRAS, 218, 453

Farrar, G. R. \& Biermann, P. L. 1998, Phys. Rev. Lett., 81, 3579

Farrar, G. R., Zaw, I., \& Berlind, A. A. 2009, arXiv :astro-ph/0904.4277

George, M. R., Fabian, A. C., Baumgartner, W. H., Mushotzky, R. F., \& Tueller, J. 2008, MNRAS, 388, L59

Gorbunov, D. S., Tinyakov, P. G., Tkachev, I. I., \& Troitsky, S. V. 2002, ApJ, 577, L93

Gorbunov, D. S., Tinyakov, P. G., Tkachev, I. I., \& Troitsky, S. V. 2004, Sov.

J. Exp. Theor. Phys. Lett., 80, 145 
Gorbunov, D. S. \& Troitsky, S. V. 2005, Astropart. Phys., 23, 175

Greisen, K. 1966, Phys. Rev. Lett., 16, 748

Gureev, S. \& Troitsky, S. 2010, Int. J. Mod. Phys. A, 25, 2917

Han, J. L. 2009, in IAU Symp.259, Cosmic Magnetic Fields: From Planets, to Stars and Galaxies, ed. K. G. Strassmeier, A. G. Kosovichev, \& J. E. Beckman(Cambridge:Cambridge Univ. Press), 455

Han, J. L. 2008, Nucl. Phys.B Proc. Supp., 175, 62

Han, J. L., Manchester, R. N.,Berkhuijsen, E. M. \&Beck, R.1997, MNRAS, 322,98

Han, J. L., Manchester, R. N., Lyne, A. G., Qiao, G. J., \& van Straten, W. 2006, ApJ, 642, 868

Han, J. L., Manchester, R. N.,\&Qiao, G. J. 1999, MNRAS, 306,371

Han, J. L. and Qiao, G. J. 1994, A\&A, 288, 759

Harari, D., Mollerach, S., \& Roulet, E. 1999, J. High Energy Phys., JHEP08(1999)022

Hardcastle, M. J., Cheung, C. C., Feain, I. J., \& Stawarz, Ł. 2009, MNRAS, 393, 1041

Hayashida, N., \& et al. 2000, astro-ph/0008102

Hillas, A. M. 1984, ARA\&A, 22, 425

Hou, L. G., Han, J. L., \& Shi, W. B. 2009, A\&A, 499, 473

Israel, F. P. 1998, A\&A Rev., 8, 237

Ivanov, A. A. 2008, Int. Cosmic Ray Conf., 4, 303

Ivanov, A. A., Knurenko, S., P., Sleptsov, I. ye. 2003, Nucl. Phys. B. Supp., 122,226

Ivanov, A. A., Krasil'Nikov, A. D., \& Pravdin, M. I. 2003, Sov. J. Exp. Theor. Phys. Lett., 78, 695

Kachelrieß, M., Ostapchenko, S., \& Tomàs, R. 2009, New J. Phys., 11, 065017

Kachelrie, M. \& Semikoz, D. V. 2006, Astropart. Phys., 26, 10

Kachelrieß, M., Serpico, P. D., \& Teshima, M. 2007, Astropart. Phys., 26, 378

Lenc, E., \& Tingay, S. J. 2009, AJ, 137, 537

Linsley, J. 1963, Phys. Rev. Lett., 10, 146

Mao, S. A., Gaensler, B. M., Haverkorn, M., Zweibel, E. G., Madsen, G. J., McClure-Griffiths, N. M., Shukurov, A., \& Kronberg, P. P. 2010, ApJ, 714, 1170

Men, H., Ferrière, K., \& Han, J. L. 2008, A\&A, 486, 819

Milgrom, M. \& Usov, V. 1995, ApJ, 449, L37

Mirabal, N., \& Oya, I. 2010, MNRAS, 405, L99

Morris, M. and Serabyn, E. 1996, ARA\&A, 34, 645

Moskalenko, I. V., Stawarz, L., Porter, T. A., \& Cheung, C. C. 2009, ApJ, 693,1261
Nagar, N. M. \& Matulich, J. 2009, arXiv: astro-ph/0912.2131

Nota,T. \& Katgert, P. 2010, A\&A, 513, A65

Pravdin, M. I., 2005, Proc. 29th ICRC, 7, 243

Piran, T. 2010, arXiv:1005.3311

Protheroe, R. J. \& Szabo, A. P. 1992, Phys. Rev. Lett., 69, 2885

Prouza, M. \& Šmída, R. 2003, A\&A, 410, 1

Rachen, J. P. \& Biermann, P. L. 1993, A\&A, 272, 161

Romero, G. E. e. 1996, Astropart. Phys., 5, 279

Ryu, D., Das, S., \& Kang, H. 2010, ApJ, 710, 1422

Sigl, G., Miniati, F., \& Ensslin, T. A. 2003, Phys. Rev. D, 68, 043002

Stanev, T. 1997, ApJ, 479, 290

Storchi-Bergmann, T., Dors, O. L., Jr., Riffel, R. A., Fathi, K., Axon, D. J., Robinson, A., Marconi, A., \&Oumlstlin, G. 2007, ApJ, 670, 959

Sun, X. H., Reich, W., Waelkens, A., \& Enßlin, T. A. 2008, A\&A, 477, 573

Takeda, M., et al 1999, ApJ, 522, 225

Takeda, M., et al, 2003, Astropart. Phys., 19, 447

Taylor, A. R., Stil, J. M., \& Sunstrum, C. 2009, ApJ, 702, 1230

The Fermi/LAT Collaboration. 2009, ApJS, 183, 46

The Pierre Auger Collaboration, 2007, Science, 318, 938

The Pierre Auger Collaboration, 2008, Astropart. Phys., 29, 188

The Pierre Auger Collaboration, 2009, arXiv:0906.2347

Tinyakov, P. G. \& Tkachev, I. I. 2001a, Sov. J. Exp. Theor. Phys. Lett., 74, 445

Tinyakov, P. G. \& Tkachev, I. I. 2001b, Sov. J. Exp. Theor. Phys. Lett., 74, 1

Tinyakov, P. G. \& Tkachev, I. I. 2002, Astropart. Phys., 18, 165

Tinyakov, P. G. \& Tkachev, I. I. 2005, Astropart. Phys., 24, 32

Torres, D. F., Reucroft, S., Reimer, O., \& Anchordoqui, L. A. 2003, ApJ, 595, L13

Véron-Cetty, M. \& Véron, P. 2006, A\&A, 455, 773

Véron-Cetty, M. \& Véron, P. 2010, A\&A, in press

Virmani, A., Bhattacharya, S., Jain, P., Razzaque, S., Ralston, J. P., \& Mckay, D. W. 2002, Astropart. Phys., 17, 489

Waelkens, A., Jaffe, T., Reinecke, M., Kitaura, F. S., \& Enßlin, T. A. 2009, A\&A, 495, 697

Waxman, E. 1995, Phys. Rev. Lett., 75, 386

Yusef-Zadeh, F. , Morris, M. \& Chance, D. 1984, Nature, 310, 557

Yusef-Zadeh, F. , Morris, M. \& Chance, D. 2004, ApJS, 155, 421

Zatsepin, G. T. \& Kuz'min, V. A. 1966, Sov. J. Exp. Theor. Phys. Lett., 4, 78 